\title{
An Efficient Semianalytical Modal Analysis of Rectangular Waveguides Containing Metamaterials with Graded Inhomogeneity
}

\author{
Qianru Weng $\mathbb{D},{ }^{1}$ Qian Lin $\mathbb{D}^{2},{ }^{2}$ and Haifeng $W u^{3}$ \\ ${ }^{1}$ Schools of Computers, Guangdong University of Technology, Guangzhou 510006, Guangdong, China \\ ${ }^{2}$ College of Physics and Electronic Information Engineer, Qinghai University for Nationalities, Xining 810007, China \\ ${ }^{3}$ Chengdu Ganide Technology, Chengdu 610073, China
}

Correspondence should be addressed to Qianru Weng; wengqianru2013@163.com

Received 16 June 2020; Revised 17 December 2020; Accepted 25 January 2021; Published 2 February 2021

Academic Editor: Hervé Aubert

Copyright $(2021$ Qianru Weng et al. This is an open access article distributed under the Creative Commons Attribution License, which permits unrestricted use, distribution, and reproduction in any medium, provided the original work is properly cited.

Rectangular waveguides containing inhomogeneous metamaterials with graded refractive-index profiles have potential applications in bending waveguides and radiation-enhanced antennas, and accurate eigenvalue solutions are prerequisite. Commonly used commercial electromagnetic solvers such as HFSS, COMSOL, and CST could not efficiently calculate the eigenvalues of waveguides containing graded refractive-index dielectrics. In this paper, an accurate and efficient semianalytical method based on the modal expansion has been proposed to solve these waveguides. The proposed method has been employed to calculate the eigenvalues, including the cutoff wavenumbers and dispersion relations, for metamaterials with various graded refractive-index profiles. Calculated results are then validated by comparison, using commercial solver HFSS, which indicates the superiority of the proposed method in accuracy and efficiency. Below-cutoff backward wave propagation is observed in waveguides filled with graded refractive-index metamaterials, which provides a new approach for waveguide miniaturization.

\section{Introduction}

Rectangular waveguides filled with inhomogeneous dielectrics are the most fundamental guided wave structures [1] to realize various functional microwave devices, such as filters [2]; couplers [3]; dividers [4]; and substrate integrated waveguides (SIWs) $[5,6]$. The overall performances of such devices strongly depend on the guided wave propagation properties of the waveguides. At the same time, the propagation properties of the waveguides depend not only on the physical dimensions of the waveguides but also on the dielectric inhomogeneity in the waveguides.

Most of the work reported in the publications before 2000 is concerned about the ordinary dielectrics (or righthanded materials, RHMs). As more and more kinds of metamaterials have been physically realized [7], especially the left-handed materials (LHMs) [8], filling rectangular waveguides with metamaterials has become a new area of research because intriguing propagation phenomena could be achieved. Propagation below the cutoff frequency in a rectangular waveguide partially filled with LHM has been theoretically analyzed and experimentally verified $[9,10]$. This phenomenon has been applied in the design of miniaturization filters and antennas. Alternately arranged righthanded materials (RHMs) and left-handed materials (LHMs) constitute a novel kind of metamaterial, which is called composite right-handed and left-handed material (CRLHM) [11]. It has been found that a rectangular waveguide filled with CRLHM supports two propagation bands: a forward wave band (also called right-handed mode) above the cutoff frequency and a backward wave band (also called left-handed mode) below the cutoff frequency $[11,12]$. A step-change in the refractive-index within the abovementioned CRLHM constitutes a basic dielectric inhomogeneity in rectangular waveguides. However, it has been already known that filling metamaterials with graded 
refractive-index profiles permits the manipulation of wave propagation in a waveguide, which could be used to achieve bending waveguides or waveguide splitting effect $[13,14]$. Thus, it is desired to acquire the propagation characteristics of a rectangular waveguide filled with CRLHM with a graded refractive-index profile.

The graded inhomogeneity of the dielectrics has severely limited the possibility to obtain the analytical eigenvalue solutions of waveguides, and accurate numerical methods need to be used. In recent years, commercial electromagnetic simulation software, including HFSS, COMSOL, and CST, are based on the full-wave numerical methods, such as the finite element method (FEM), the finite-difference time-domain (FDTD), the method of moments (MoM), and the boundary element method (BEM) [15, 16]. Although these electromagnetic solvers have powerful analysis capabilities, they require a very long calculation time and a large amount of computer memory for structures containing complex inhomogeneous dielectrics. Most of the solvers cannot even directly analyze the structures containing gradual spatially varying dielectrics, for example, metamaterials with graded refractive-index profiles.

In this paper, an accurate semianalytical method based on a one-dimensional modal expansion theory has been proposed to calculate the eigenvalues of rectangular waveguides filled with CRLHM having graded refractive-index profiles. A similar method has been used to obtain the eigenvalue solutions for dielectric slab waveguides with arbitrary dielectric profiles [17] and optical graded-index planar waveguides with metal cladding [18]. This method is derived from the application of the fundamental Maxwell equations and expands the unknown electromagnetic field into a complete set of orthogonal functions, which leads to a matrix eigenvalue problem whose solution provides all the necessary eigenvalues. The eigenvalues can be applied to obtain the cutoff and dispersion characteristics. The general formulations of the proposed method are described in Section 2. Complete modal analyses, including the longitudinal-section electric (LSE) and longitudinal-section magnetic (LSM) modes, for rectangular waveguides filled with CRLHM having several representative graded refractive-index profiles are conducted using the proposed method in Section 3. Several interesting phenomena, including the less intuitive cutoff dependence on dielectric parameters and the below-cutoff propagation characteristics, are also presented in this section. The accuracy and efficiency of the proposed method are validated by comparison, using the commercial simulation software HFSS in Section 4 . Section 5 concludes the development potential of the proposed method and phenomena.

\section{Theoretical Formulation}

Figure 1 shows the considered rectangular waveguide filled by composite right-handed and left-handed metamaterial (CRLHM) with a graded refractive-index profile. Such waveguide is homogeneous along the $z$-axis, and the wave propagation is also along the $z$-axis. Therefore, the electromagnetic fields have an $\mathrm{e}-\mathrm{j} \beta \mathrm{z}$ dependence, and $\beta$ is the propagation constant. The time-harmonic has an ejwt dependence. The Maxwell equations are rearranged in a source-free region.

$$
\begin{aligned}
\Delta \times \Delta \times \vec{E}-\omega^{2} \mu(\omega, x) \varepsilon(\omega, x) \vec{E} & =\frac{\Delta \mu(\omega, x)}{\mu(\omega, x)} \times \Delta \times E, \\
\Delta \times \Delta \times \vec{H}-\omega^{2} \mu(\omega, x) \varepsilon(\omega, x) \vec{H} & =\frac{\Delta \varepsilon(\omega, x)}{\varepsilon(\omega, x)} \times \Delta \times \vec{H}, \\
\Delta \cdot[\varepsilon(\omega, x) \vec{E}] & =\left\{\frac{\partial}{\partial x}\left[\varepsilon(\omega, x) E_{x}\right]+\varepsilon(\omega, x) \frac{\partial}{\partial y} E_{y}-j \beta \varepsilon(\omega, x) E_{z}\right\}=0, \\
\Delta \cdot[\mu(\omega, x) \vec{H}] & =\left\{\frac{\partial}{\partial x}\left[\mu(\omega, x) H_{x}\right]+, \mu(\omega, x) \frac{\partial}{\partial y} H_{y}-j \beta \mu(\omega, x) E_{z}\right\}=0,
\end{aligned}
$$

where the permittivity $\epsilon(\omega, x)$ and the permeability $\mu(\omega, x)$ are the frequency-dependent dielectric permittivity and magnetic permeability, respectively.

\subsection{The Definition of CRLHM with a Graded Refractive-Index} Profile. The permittivity and the permeability of a CRLHM with a graded refractive-index profile can be written as

$$
\begin{aligned}
& \epsilon(\omega, x)=\epsilon_{0} \epsilon_{r}(\omega, x)=\varepsilon_{0} \varepsilon_{r}(\omega)[1-h(x)], \\
& \mu(\omega, x)=\mu_{0} \mu_{r}(\omega, x)=\mu_{0} \mu_{r}(\omega)[1-h(x)],
\end{aligned}
$$

where $\varepsilon r(\omega)$ and $\mu r(\omega)$ are the frequency-dependent relative permittivity and permeability, which can be described by Drude or Lorentz models [7]; $\varepsilon 0$ and $\mu 0$ are the free space permittivity and permeability; and $h(x)$ is a spatial-dependent profile function, which can be arbitrary. The sketches of profile functions of graded refractive-index CRLHMs are presented in Figure 2, showing a gradual transition from RHM to LHM [19]. Here, the permittivity $\epsilon(\omega, x)$ and the permeability $\mu(\omega, x)$ are assumed to have the identical profile function.

In this paper, three kinds of profile functions $h(x)$ are under consideration: 


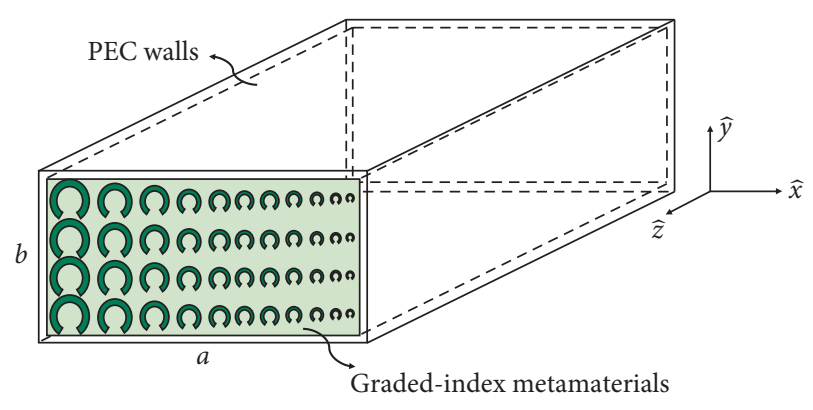

FIGURE 1: Rectangular waveguide filled with a metamaterial with a graded refractive-index profile.

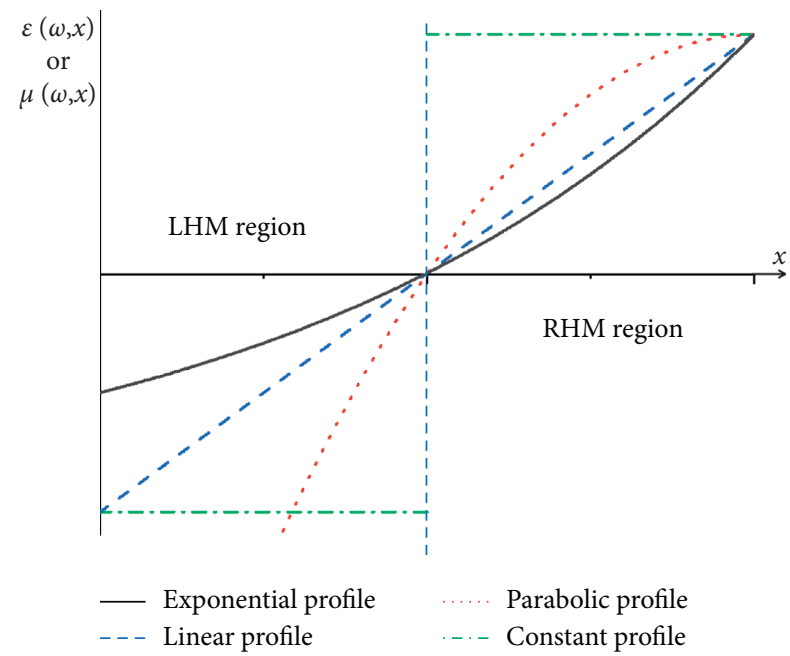

FIGURE 2: Gradual spatially varying effective permittivity $\epsilon(\omega, x)$ and permeability $\mu(\omega, x)$ of CRLHM with exponential, linear, parabolic, and constant profile functions.

$$
h(x)=\left\{\begin{array}{l}
\mathrm{d} x, \text { linear profile function, } \\
\mathrm{d} x^{2}, \text { parabolic profile function, } \\
\exp (-\mathrm{d} x), \text { exponential profile function, }
\end{array}\right.
$$

where $d$ is a positive parameter describing the steepness of the transition from RHM to LHM within a CRLHM. If $d=0$, the graded refractive-index CRLHM degenerates into the aforesaid step-changed CRLHM. This specific case will be discussed in the following.

2.2. Hybrid LSE and LSM Modes Definition. Standard propagation modes in a rectangular waveguide filled with an inhomogeneous metamaterial as shown in Figure 1 are not, in general, either a transverse electric (TE) or a transverse magnetic (TM) mode but a hybrid of TE and TM modes. This suggests that the hybrid modes in such waveguide may be derived from magnetic or electric types of Hertzian potential functions, having single components directed normal to the inhomogeneous direction ( $x$-axis).
(1) From the magnetic Hertzian potential, the solution for a mode that has no component of the electric field normal to the $x$-axis is obtained. The electric field thus lies in the longitudinal interface (yz-plane) plane, and this mode is referred to as a longitudinalsection electric (LSE) mode [1].

$$
\vec{\Pi}_{h}=\vec{a}_{x} \phi_{h}(x, y) e^{-j \beta z} .
$$

(2) From the electric Hertzian potential, the solution for a mode with no magnetic field component normal to the $x$-axis is obtained. This mode is called a longitudinal-section magnetic (LSM) mode [1].

$$
\vec{\Pi}_{e}=\vec{a}_{x} \phi_{e}(x, y) e^{-j \beta z} .
$$

\subsection{Electromagnetic Fields and Boundary Conditions}

2.3.1. LSE Modes. In a rectangular waveguide filled with inhomogeneous metamaterials, as shown in Figure 1, the whole fields of the LSE mode can be derived from $E_{y}$ [1]. The $y$-component of Helmholtz's equation is

$$
\left\{\nabla \mu(\omega, x) \nabla \times\left[\frac{1}{\mu(\omega, x) \nabla \times \vec{E}}\right]\right\} y+\omega^{2} \mu(\omega, x) \vec{E}_{y}=0 .
$$

The electric field $E_{y}$ could be written as

$$
E_{y}=\phi_{h}(x) \cos \frac{l \pi y}{b} e^{-j \beta z}
$$

For LSE modes, $E_{y}$ shall vanish on the perfect conducting boundaries $(x=0, a)$ of the rectangular waveguide. Thus, the potential function $\phi h(x)$ satisfies the boundary condition:

$$
\left.\phi_{h}(x)\right|_{x=0, a}=0 .
$$

2.3.2. LSM Modes. Similarly, the whole fields of the LSM mode can be derived from $H_{y}$ [1]. The y-component of Helmholtz's equation is

$$
\left\{\nabla \in(\omega, x) \nabla \times\left[\frac{1}{\epsilon(\omega, x) \nabla \times \vec{H}}\right]\right\} y+\omega^{2} \mu(\omega, x) \in(\omega, x) \vec{H}_{y}=0 .
$$

The magnetic field $H_{y}$ could be written as

$$
H_{y}=\phi_{e}(x) \sin \frac{l \pi y}{b} e^{-j \beta z}
$$

For LSM modes, $\partial H_{y} / \partial x$ shall vanish on the perfecting conducting boundaries of the rectangular waveguide at $x=0$, a. Thus, the potential function $\phi e(x)$ satisfies the boundary condition:

$$
\left.\frac{\mathrm{d} \phi_{e}}{\mathrm{~d} x}\right|_{x=0, a}=0 .
$$




\subsection{The Semianalytical Method Based on a Modal Expansion \\ Theory}

2.4.1. LSE Modes. Substituting equation (7) into equation (6), the potential function $\phi h(x)$ for the LSE mode satisfies the following equation:

$$
\frac{\mathrm{d}^{2} \phi h(x)}{\mathrm{d} x^{2}}-\frac{\mathrm{d} \ln \mu(\omega, x)}{\mathrm{d} x} \frac{\mathrm{d} \phi h(x)}{\mathrm{d} x}+\left[\omega^{2} \mu(\omega, x) \in(\omega, x)-\beta^{2}-\left(\frac{l \pi}{b}\right)^{2}\right] \phi h(x)=0 .
$$

Equation (12) and the boundary condition (8) constitute a Sturm-Liouville system [1]. According to the modal expansion theory, $\phi h(x)$ could be expanded as a complete set of orthogonal functions.

$$
\phi h(x)=\sum_{m=1}^{N} A_{m} g_{m}(x)
$$

where $A_{m}$ is the amplitude. For the LSE mode, a suitable orthogonal function $g_{m}(x)$ is chosen [1].

$$
g_{m}(x)=\sin \frac{m \pi x}{a} ; \quad m=1,2,3, \ldots
$$

As shown in equation (13), a finite series (N) is used in the derivation of eigenequation. The derivation process is as follows.

Firstly, substitute equation (13) into equation (12), and then multiply the resulting equation by the orthogonal function $\sin n \pi x / a(n=1,2,3, \ldots)$;

Secondly, integrating over the waveguide width a, equation (12) is transformed to the following:

$$
-\left[\beta^{2}+\left(\frac{l \pi}{b}\right)^{2}\right] A_{n}+\sum_{m=1}^{N} A_{m} \int_{0}^{a}\left[\frac{\mathrm{d} \ln \mu(\omega, x)}{\mathrm{d} x} \frac{\mathrm{d} g_{m}}{\mathrm{~d} x} g_{n}\right] \mathrm{d} x+\sum_{m=1}^{N} A_{m} \int_{0}^{a} \omega^{2} \mu(\omega, x) \in(\omega, x) g_{m} g_{n} \mathrm{~d} x=0
$$

Thirdly, equations (15) can be written as a matrix form as follows:

$$
[\mathrm{P}][\mathbf{A}]=0 \text {, for LSE mode, }
$$

$$
\begin{aligned}
p_{m m} & =-\left[\beta^{2}+\left(\frac{l \pi}{b}\right)^{2}\right]+\int_{0}^{a}\left[\frac{\mathrm{d} \ln \mu(\omega, x)}{\mathrm{d} x} \frac{\mathrm{d} g_{m}}{\mathrm{~d} x} g_{m}\right] \mathrm{d} x+\int_{0}^{a} \omega^{2} \mu(\omega, x) \in(\omega, x) g_{m}^{2} \mathrm{~d} x(m=n), \\
p_{m n} & =p_{n m}=\int_{0}^{a}\left[\frac{\mathrm{d} \ln \mu(\omega, x)}{\mathrm{d} x} \frac{\mathrm{d} g_{m}}{\mathrm{~d} x} g_{n}\right] \mathrm{d} x+\int_{0}^{a} \omega^{2} \mu(\omega, x) \in(\omega, x) g_{m} g_{n} \mathrm{~d} x(m \neq n) .
\end{aligned}
$$

Finally, the eigenequation of LSE modes is obtained:

$$
\operatorname{Det}[\mathbf{P}]=0 \text {. }
$$

The accuracy of the calculated eigenvalues is closely related to the value of the series number $\mathrm{N}$ of the modal expansion in equation (13), and the higher the value of $\mathrm{N}$, the more accurate eigenvalues could be obtained through this method. However, the larger the value of $\mathrm{N}$, the higher the order of the matrix $\mathbf{P}$, and the greater amount of calculation is required to solve the determinant of the matrix $\mathbf{P}$ as shown in equation (18). In this paper, the eigenvalue accuracy is characterized by using the change of where A presents a column vector with elements $A_{\mathrm{m}}$ and $\mathbf{P}$ is a matrix whose elements are defined as follows: the eigenvalues, $\Delta \beta=|1-\beta N \beta N-1| \%$. Through precalculation, it has been found that for rectangular waveguides filled with CRLHM with constant, linear, parabolic, and exponential profiles, $\Delta \beta=0.1 \%$ of the LSE mode could be achieved by using $N=40$ series number in general. In this paper, the series number $\mathrm{N}$ is chosen as 40 to calculate the eigenvalues of the LSE mode.

2.4.2. LSM Modes. Substituting equation (10) into equation (9), the potential function $\phi e(x)$ for the LSM mode satisfies the following equation: 


$$
\frac{\mathrm{d}^{2} \phi_{e}(x)}{\mathrm{d} x^{2}}-\frac{\mathrm{d} \ln \in(\omega, x)}{\mathrm{d} x} \frac{\mathrm{d} \phi_{e}(x)}{\mathrm{d} x}+\left[\omega^{2} \mu(\omega, x) \in(\omega, x)-\beta^{2}-\left(\frac{l \pi}{b}\right)^{2}\right] \phi_{e}(x)=0 .
$$

The eigenequation derivation of the LSM mode follows the same line as that of the LSE mode described above. Considering the boundary condition for the LSM mode, $\phi e x$ takes the following form:

$$
\phi_{e}(x)=\sum_{m=0}^{N} B_{m} f_{m}(x)
$$

$$
f_{m}(x)=\cos \frac{m \pi x}{a} ; \quad m=0,1,2,3, \ldots
$$

For the LSM mode, substituting equation (20) into equation (19), then multiplying the resulting equation by the orthogonal function $\cos n \pi x a(n=0,1,2,3, \ldots)$ for the LSM mode, and then integrating over the waveguide width a, equation (19) is transformed to

For the LSM mode, a suitable function $f_{m}(x)$ is chosen as follows [1]:

$$
-\beta^{2}+\left(\frac{l \pi}{b}\right)^{2} B_{n}+\sum_{m=1}^{N} B_{m} \int_{0}^{a}\left[\frac{\mathrm{d} \ln [\varepsilon(\omega, x)]}{\mathrm{d} x} \frac{\mathrm{d} f_{m}}{\mathrm{~d} x} f_{n}\right] \mathrm{d} x+\sum_{m=1}^{N} B_{m} \int_{0}^{a} \omega^{2} \mu(\omega, x) \in(\omega, x) f_{m} f_{n} \mathrm{~d} x=0
$$

Equation (22) can be written as a matrix form:

$$
[\mathbf{Q}][\mathbf{B}]=0 \text {, for LSM mode, }
$$

where $\mathbf{B}$ presents a column vector with elements $B_{\mathrm{m}}$ and $\mathbf{Q}$ is a matrix whose elements are defined as

$$
\begin{aligned}
& q_{m m}=-\left[\beta^{2}+\left(\frac{l \pi}{b}\right)^{2}\right]+\int_{0}^{a}\left[\frac{\mathrm{d} \ln [\varepsilon(\omega, x)]}{\mathrm{d} x} \frac{\mathrm{d} f_{m}}{\mathrm{~d} x} f_{m}\right] \mathrm{d} x+\int_{0}^{a} \omega^{2} \mu(\omega, x) \in(\omega, x) f_{m}^{2} \mathrm{~d} x(m=n), \\
& q_{m n}=q_{n m}=\int_{0}^{a}\left[\frac{\mathrm{d} \ln [\varepsilon(\omega, x)]}{\mathrm{d} x} \frac{\mathrm{d} f_{m}}{\mathrm{~d} x} f_{n}\right] \mathrm{d} x+\int_{0}^{a} \omega^{2} \mu(\omega, x) \in(\omega, x) f_{m} f_{n} \mathrm{~d} x(m \neq n) .
\end{aligned}
$$

Thus, the eigenfunction for LSM modes of a rectangular waveguide filled inhomogeneous metamaterials can be written as

$$
\operatorname{Det}[\mathbf{Q}]=0 .
$$

Therefore, the cutoff frequencies and the dispersion relations of the LSM modes could be obtained by calculating eigenvalues of the matrix $\mathbf{Q}$. The accuracy of the calculated eigenvalues of the LSM modes is also closely related to the series number $\mathrm{N}$ of the modal expansion in equation (20). From the precalculation, to obtain an error within $\Delta \beta=0.1 \%$ for the LSM modes, $N=50$ series number needs to be chosen to satisfy this accuracy requirement. Thus, in this paper, the series number $\mathrm{N}$ is chosen as 50 to calculate the eigenvalues of the LSM mode.

2.5. A Specific Case: A Rectangular Waveguide Filled with Homogeneous Dielectric. For $h(x)=0$, a rectangular waveguide filled with graded-index dielectric degenerates into a rectangular waveguide filled with homogeneous dielectric. Thus, the dielectric parameters are changed into

$$
\begin{aligned}
\varepsilon(\omega) & =\varepsilon_{0} \varepsilon_{r}(\omega), \\
\mu(\omega) & =\mu_{0} \mu_{r}(\omega) .
\end{aligned}
$$

Substitute equation (26) into the dispersion equations (18) and (25) for LSE and LSM modes. For these two kinds of modes, the dispersion equation becomes

$$
\begin{aligned}
& \beta= \pm k_{0} \sqrt{\varepsilon_{r}(\omega) \mu_{r}(\omega)\left[1-\left(\frac{k_{c}}{k_{0}}\right)^{2}\right]}, \\
& k_{c}=\sqrt{\left(\frac{m \pi}{a}\right)^{2}+\left(\frac{n \pi}{b}\right)^{2}},
\end{aligned}
$$

which corresponds precisely to the definition of the dispersion constants for TE and TM modes in a homogeneous waveguide.

\section{Results and Discussion}

From the waveguide modal theory presented in the previous section, a specific code that can compute the eigenvalues of a rectangular waveguide filled with a graded-index CRLHM 


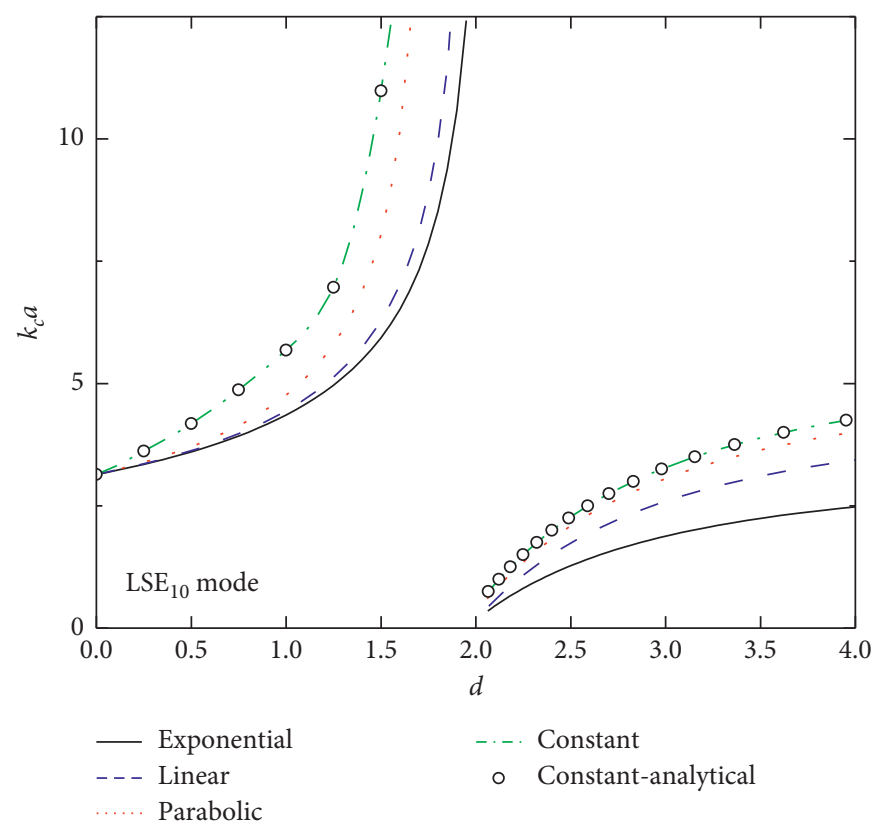

(a)

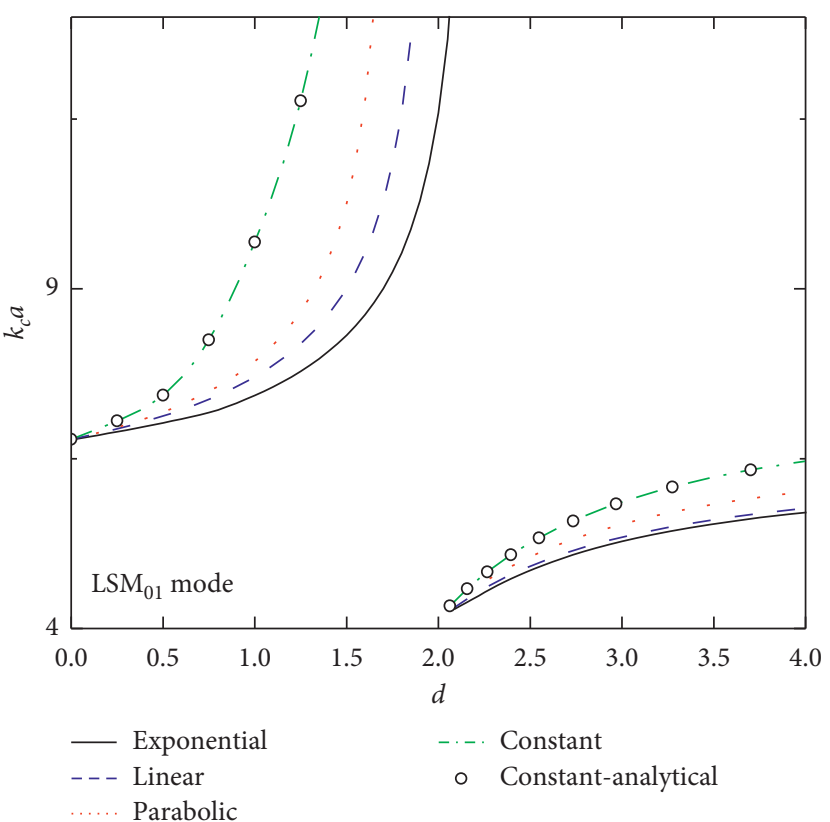

(b)

FIGURE 3: The normalized cutoff wavenumbers $k_{c} a$ versus the profile parameter $\mathrm{d}$ for various graded-index profile functions, $a=1, b=0.5$ : (a) $\mathrm{LSE}_{10}$ mode and (b) $\mathrm{LSM}_{01}$ mode.

has been developed. In this section, the cutoff characteristics and dispersion diagrams are obtained from the calculated eigenvalues. In order to extend the results for a general use, the solution data in this paper are normalized for all length scales.

3.1. CutoffCharacteristics. The fundamental LSE mode in an inhomogeneous rectangular waveguide is the $\mathrm{LSE}_{10}$ mode, while the fundamental LSM mode is the $\mathrm{LSM}_{01}$ mode. The relationships between the normalized cutoff wavenumber $k_{c} a$ and the profile parameter $\mathrm{d}$ for the $\mathrm{LSE}_{10}$ mode and $\mathrm{LSM}_{01}$ mode are shown in Figures 3(a) and 3(b), respectively. Notably, $\mathrm{d}$ represents the slope of the profile function. The higher the value of $d$ is, the larger the area is occupied by LHM. The representative waveguide has physical dimensions $a=1$ and $b=0.5$.

As shown in Figure 3(a), when $d=0$, the rectangular waveguide is filled with a homogeneous RHM. Thus, the normalized cutoff wave number of the $\mathrm{LSE}_{10}$ mode is $k_{c} a=3.14$. In the region of $\mathrm{d}<2$, RHM occupies a larger area, and $k_{c} a$ increases as the value of $d$ increases (i.e., the occupied area of RHM increases). This behavior follows the well-known result that the cutoff wavenumber of a rectangular waveguide increases as the filling area of the RHM decreases [1].

In the region of $d>2$, the graded-index LHM occupies the larger area, and a larger profile parameter $d$ in this range produces an increased $k_{c} a$; as $\mathrm{d} \longrightarrow 2, k_{c} a$ of $\mathrm{LSE}_{10}$ mode is dramatically lowered which is very different from the cutoff dependence of RHM, suggesting that this type of rectangular waveguide may support propagation at very low wavenumbers.
In Figure 3(a), the results for constant, linear, parabolic, and exponential profile functions are presented. The shapes of these curves are similar in general. A constant profile, which means filling a rectangular waveguide with homogeneous RHM and homogeneous LHM (abrupt transitions between LHM and RHM), has exact analytical solutions [11]. In Figure 3(a), for the constant profile, the approximate solution using the proposed technique (green dot-dash line) is compared with the exact analytical solution (empty black circles). These two solutions agree well with each other. In Figure 3(b), the relationship between the normalized cutoff wavenumbers $k_{c} a$ versus $\mathrm{d}$ for the $\mathrm{LSM}_{01}$ modes is presented.

In the region of $d \longrightarrow 2$, the cutoff wavenumbers can be dramatically lowered, and the transversal dimension of the designed rectangular waveguides working in this region can be extremely small. Thus, operation in these frequency-reduced regions of the $\mathrm{LSE}_{10}$ and $\mathrm{LSM}_{01}$ modes offers the potential for the miniaturization of rectangular waveguide components.

\subsection{Dispersion Relations}

3.2.1. LSE Modes. For the chosen waveguide dimensions, $a=1$ and $b=0.5$, it can be observed from Figure 3(a) that a profile parameter of $\mathrm{d}=2.1$ will result in reduced normalized cutoff wavenumbers $k_{c} a=0.45,0.51,0.75$, and 0.92 for the exponential, linear, parabolic, and constant profiles of the $\mathrm{LSE}_{10}$ mode, respectively. In Figure 4, the dispersion relations for various profile functions of the fundamental $\mathrm{LSE}_{10}$ mode and the higher-order $\mathrm{LSE}_{20}$ mode are presented. Here, the curves are represented by different symbols, indicating the 


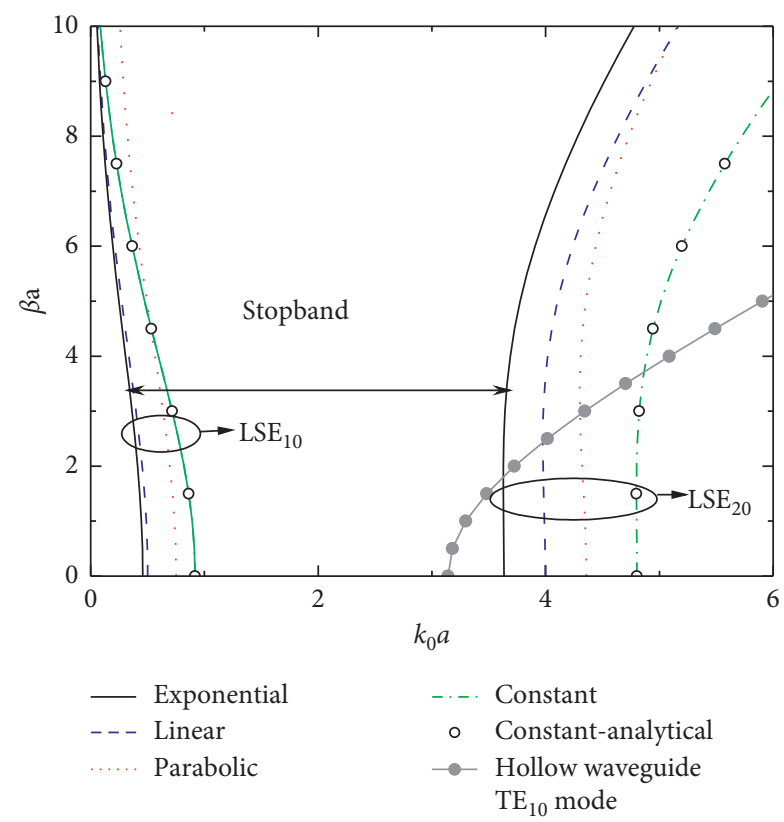

FIgURE 4: The dispersion relations of the CRLHM-filled rectangular waveguide's LSE modes for exponential, linear, parabolic, and constant profiles and the dispersion relation of the $T E_{10}$ modes of a hollow rectangular waveguide with the same dimension, $d=2.1$.
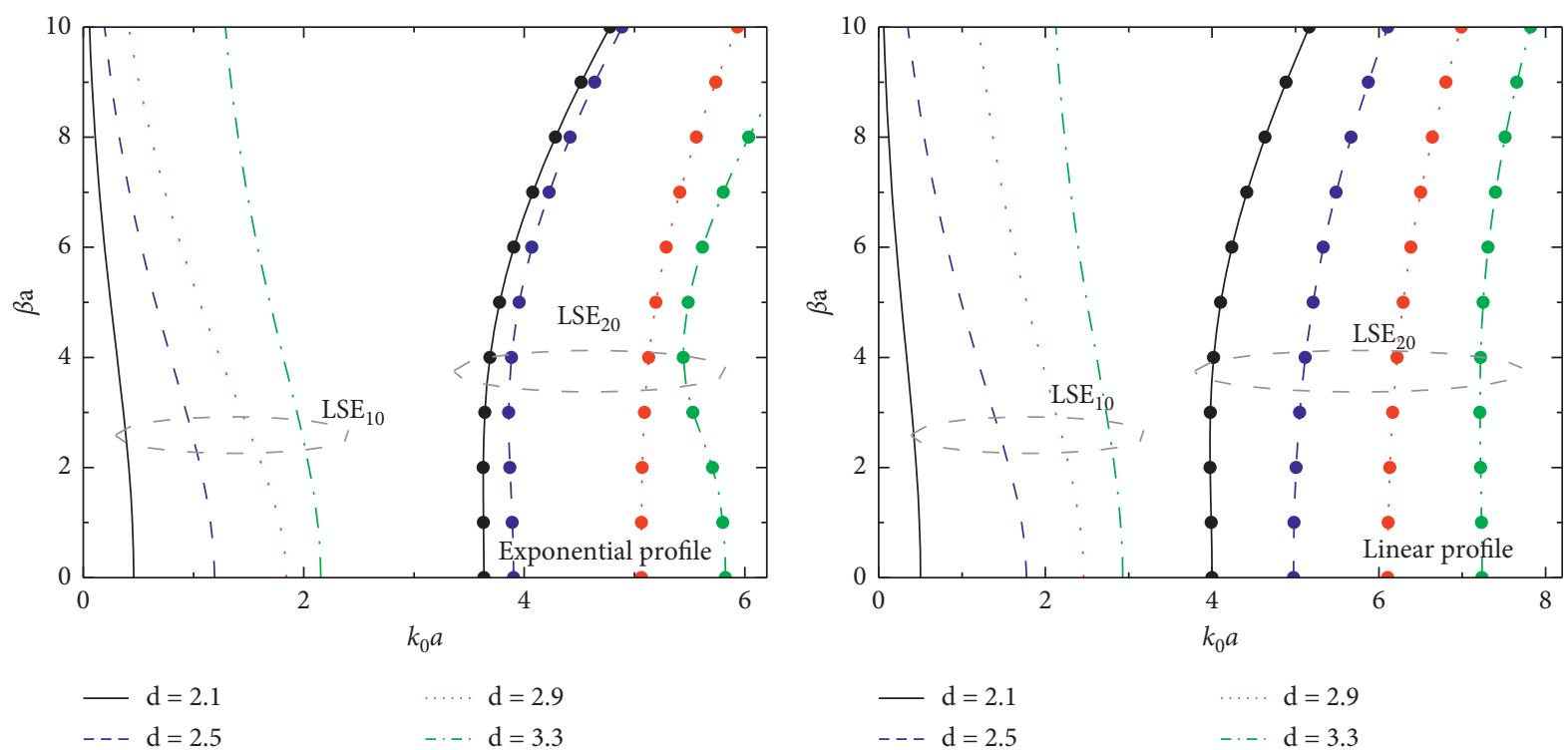

(a)

(b)

Figure 5: Continued. 


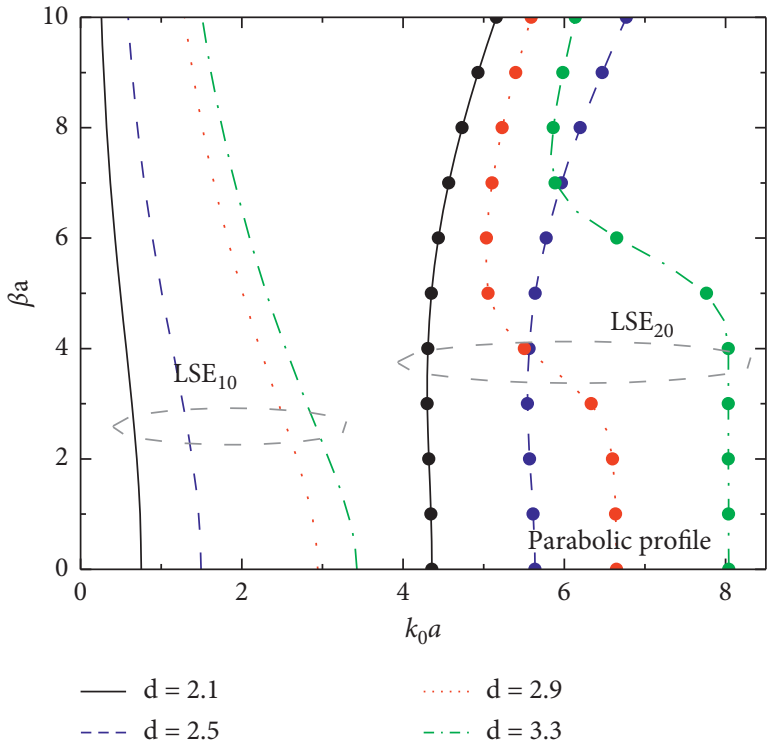

(c)

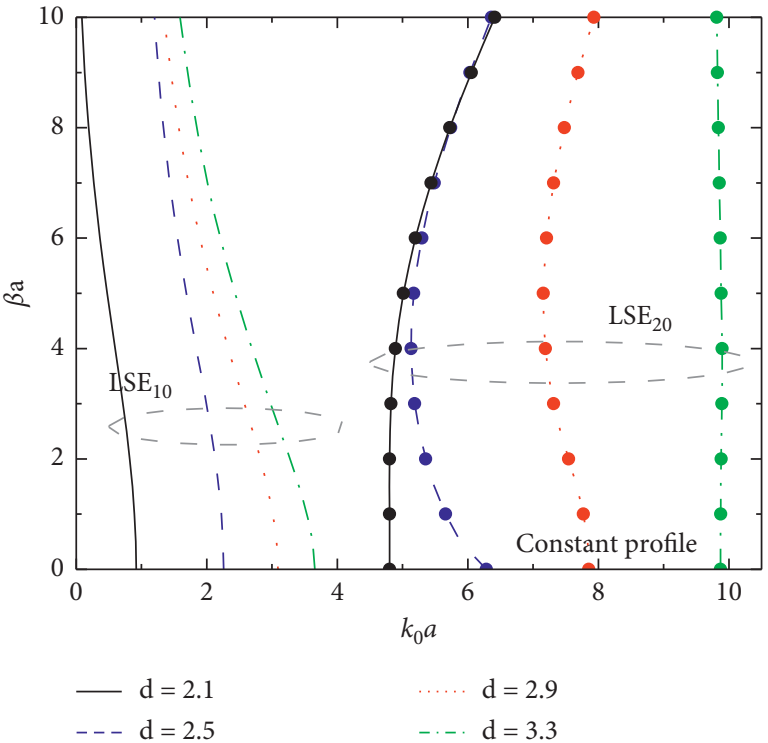

(d)

Figure 5: The dispersion relations of the LSE modes with various values of the profile parameter $\mathrm{d}$ and the step width of $\Delta \mathrm{d}=0.4$ : $(\mathrm{a})$ exponential profile; (b) linear profile; (c) parabolic profile; (d) constant profile.

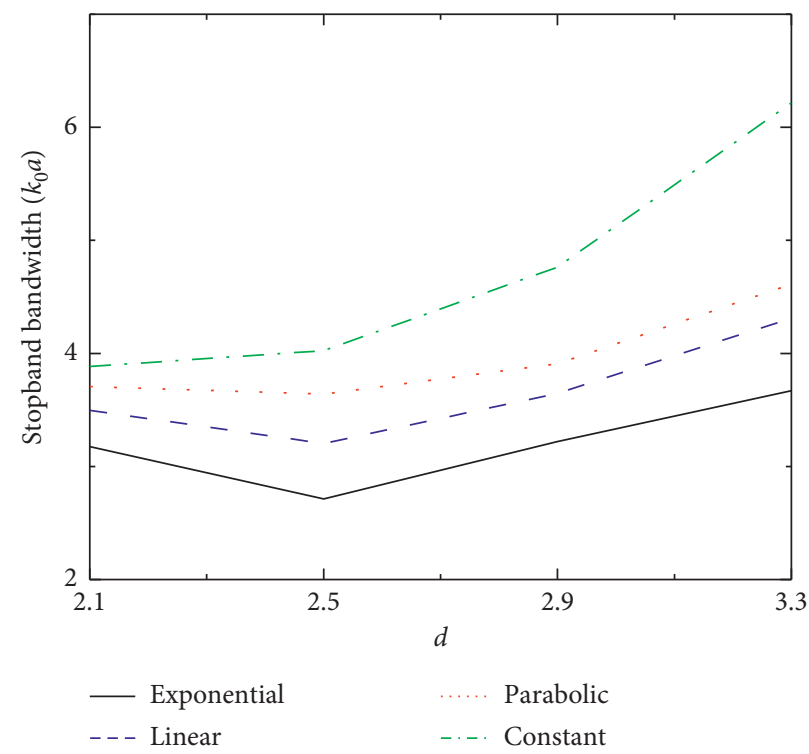

FIgURE 6: The stopband bandwidths between the backward $\mathrm{LSE}_{10}$ mode and the forward $\mathrm{LSE}_{20}$ mode versus the profile parameter $\mathrm{d}$ for the constant, linear, parabolic, and exponential profiles.

normalized propagation characteristics $\beta a$ for different profiles. The $\mathrm{TE}_{10}$ mode dispersion relation of a hollow rectangular waveguide with the same dimensions is also shown for comparison, where the dispersion curve is represented using solid gray circles. Interestingly, the rectangular waveguide filled with graded-index CRLHM supports two propagation bands: a forward wave band $(\partial \omega / \partial \beta>0)$ and a back wave band $(\partial \omega / \partial \beta>0)$ below the cutoff frequency. There is a stopband between these two propagation bands, which is influenced by the profile functions. The backward wave propagation band is significantly below the fundamental $\mathrm{TE}_{10}$ mode of a hollow rectangular waveguide. The analytical dispersion solutions have been calculated for a rectangular waveguide filled by the CRLHM with a constant profile, as indicated in Figure 4 , and good agreement is observed between the analytical solution and the approximate solution presented in this paper.

It is known that the dispersion relations of the guided waves are dependent on the profile parameter d. In Figure 5, 


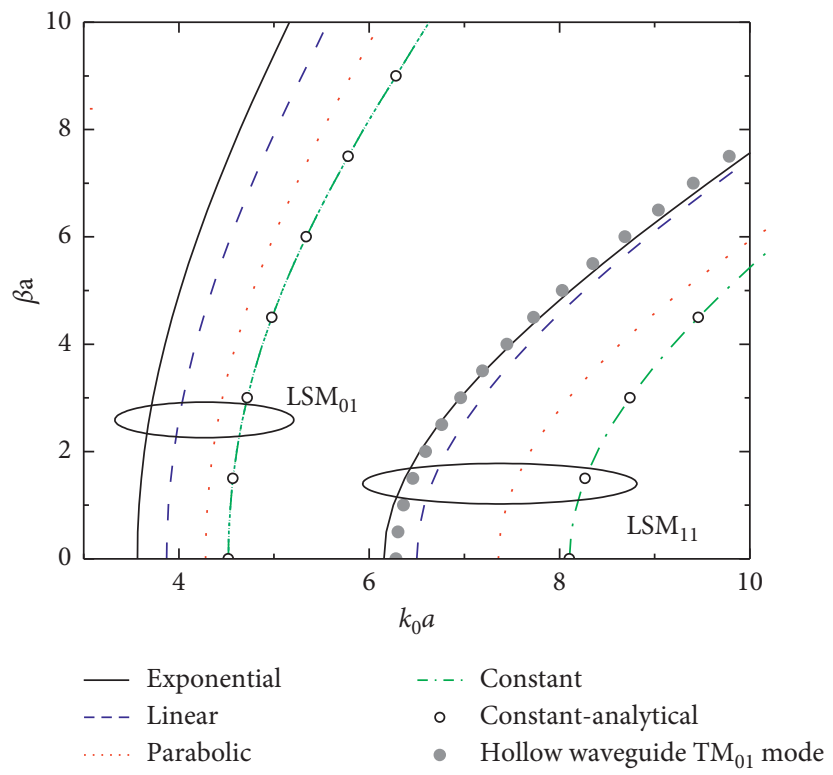

FIgURE 7: The dispersion relations of LSM modes for constant, linear, parabolic, and exponential profiles and the dispersion of the TM $\mathrm{T}_{01}$ modes of a hollow rectangular waveguide with the same dimension.

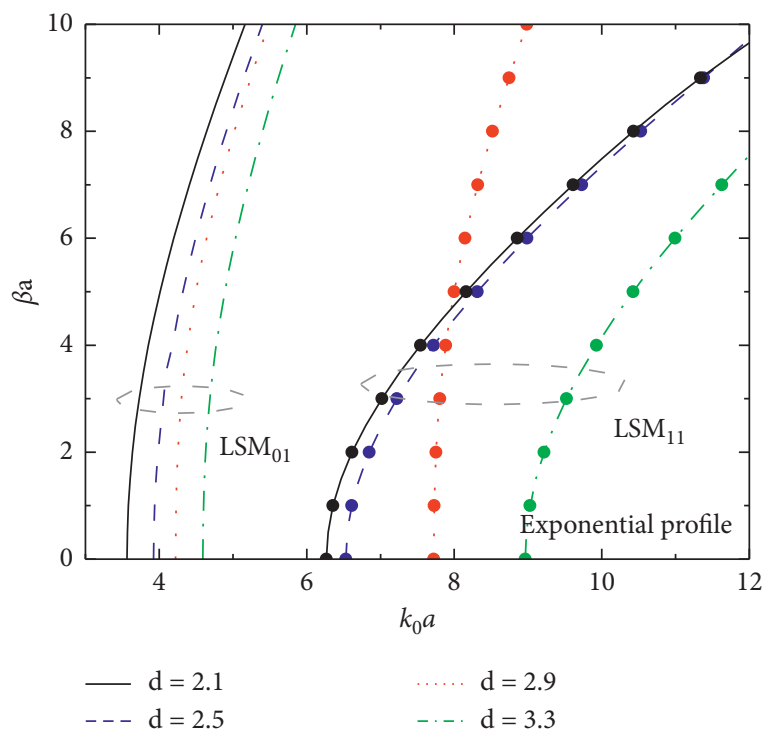

(a)

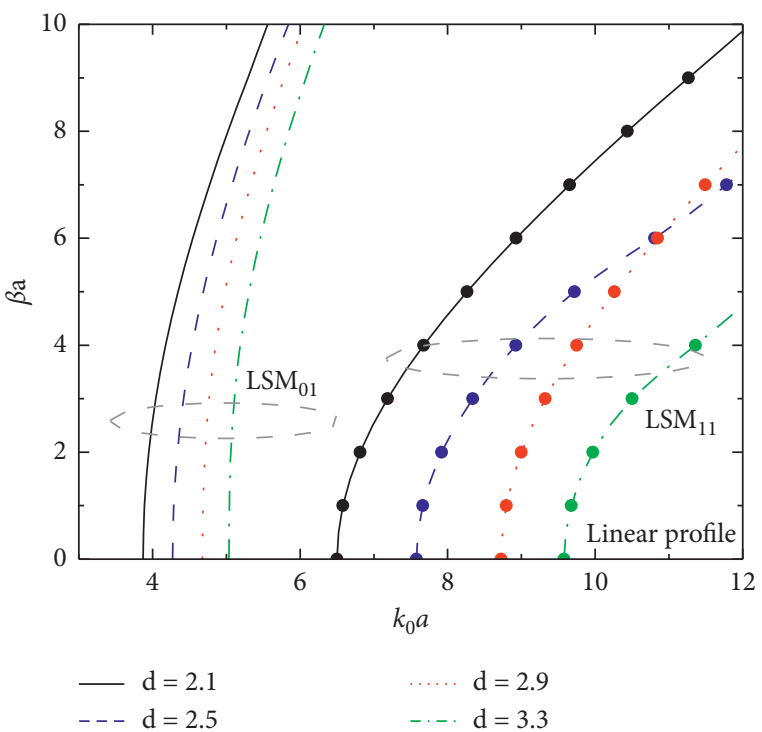

(b)

Figure 8: Continued. 


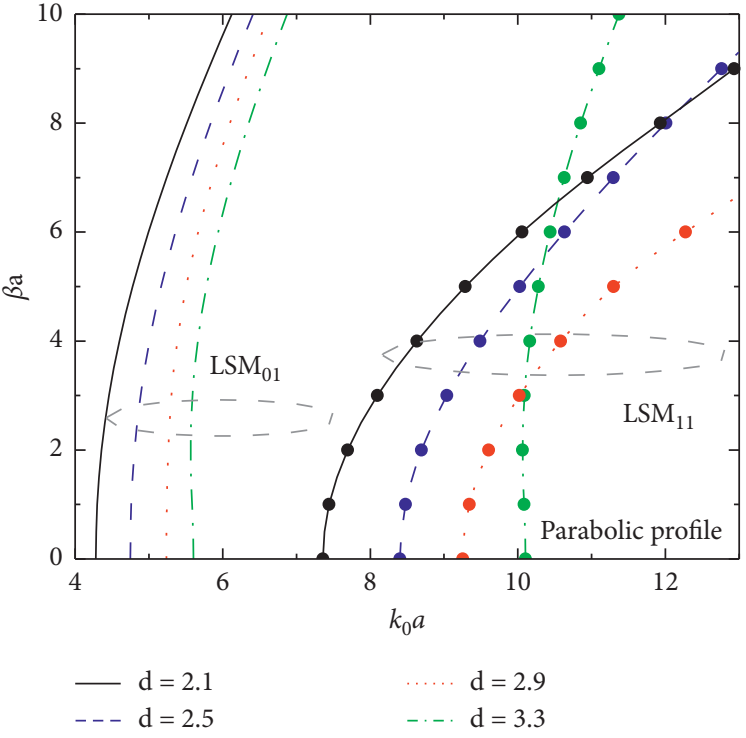

(c)

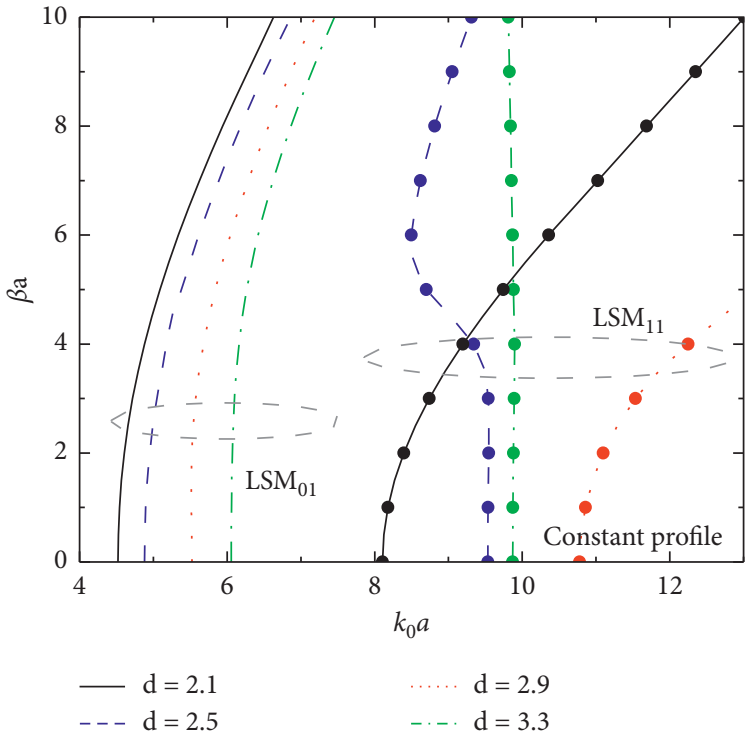

(d)

Figure 8: The dispersion relations of the LSM modes with various values of the profile parameter $\mathrm{d}$ and step width of $\Delta \mathrm{d}=0.4$ : $(\mathrm{a})$ exponential profile; (b) linear profile; (c) parabolic profile; (d) constant profile.

the dispersion relations of the fundamental $\mathrm{LSE}_{10}$ mode and the higher-order $\mathrm{LSE}_{20}$ mode are shown for the exponential, linear, parabolic, and constant profile functions in the region where the value of $d$ is near the frequency-reduced region. In Figure 5, backward wave propagation of the $\mathrm{LSE}_{10}$ mode below the cutoff frequency could be observed for the four profile functions, while the $\mathrm{LSE}_{20}$ modes support forward wave propagation. Some dispersion curves of the $\mathrm{LSE}_{20}$ modes in Figure 5, such as the dispersion curve for $d=3.3$ of the exponential profile and the dispersion curves for $\mathrm{d}=$ 2.9 and 3.3 of the parabolic profile, exhibit negative group velocities $((\partial \omega / \partial \beta)<0)$, which could be observed in metallic waveguides containing metamaterials [11].

From Figure 5, it can be found that the bandwidths of the stopband are affected by the profile functions and the profile parameter $\mathrm{d}$. To clearly illustrate the parameter dependence of the stopband bandwidths, Figure 6 shows the stopband bandwidths versus the profile parameter $d$ for different profile functions: constant, linear, parabolic, and exponential. The results show that the constant profile has the widest stopband bandwidth and increases as the parameter $\mathrm{d}$ increases. For the exponential, linear, and parabolic profiles, the bandwidths decrease as $d$ increases at first and then increase as $d$ increases.

3.2.2. LSM Modes. Figure 7 presents the dispersion relations of the fundamental $\mathrm{LSM}_{01}$ mode and the higher-order $\mathrm{LSM}_{11}$ mode for various profile functions when $d=2.1$. The $\mathrm{TM}_{01}$ mode dispersion of a hollow rectangular waveguide of the same dimension is also plotted for comparison. Both of $\mathrm{LSM}_{01}$ and $\mathrm{LSM}_{11}$ modes propagate forward $((\partial \omega / \partial \beta)>0)$, and the curve slope of the $\mathrm{LSM}_{01}$ mode is larger than that of the $\mathrm{LSM}_{11}$ mode. The cutoff wavenumbers of the higher-order $\mathrm{LSM}_{11}$ mode in the CRLHM-filled rectangular waveguide are near the cutoff wavenumber of the $\mathrm{TM}_{01}$ mode of a hollow rectangular waveguide. Figure 8 shows the dispersion relations for different profile functions versus different values of $\mathrm{d}$. The negative group velocities $((\partial \omega / \partial \beta)>0)$ could be observed in a constant profile.

\section{Full-Wave Simulation Validation}

Validation of the proposed numerical method has been conducted by the commercial finite element method (FEM) solver HFSS. HFSS is based on the discretization of the electromagnetic structures which has very high analysis capability, but the discretization is very time- and memoryconsuming. Since the spatial-dependent permittivity and permeability for the graded-index metamaterials cannot be set in HFSS, discretization on the graded-index metamaterial is conducted. As shown in the inset of Figure 9, the studied region $[0, a]$ is divided into $n$ subregions, and the subregion length is $x_{i}-x_{i-1}$. The division of subintervals can be equal or unequal. If the value of $n$ is large enough, the permittivity and the permeability of each subinterval can be viewed as constants. The constants could be replaced by the left endpoint's values, the right endpoint, or the midpoint. Here, the values of the right endpoints are employed.

The accuracy of the HFSS simulation results is directly related to the number of the discretized subareas for the graded-index metamaterials. Convergence analysis for the subregion number is shown in Figure 9 for a metamaterial with a parabolic profile. The horizontal axis is the iteration step number, and the vertical axis is the change in the propagation constant $\beta$, which is defined as $\Delta \beta=\left|1-\left(\beta_{s} / \beta_{s-1}\right)\right| \%$. For each iteration, the length of the subinterval is half of the previous iteration. The starting 




Figure 9: Convergence analysis conducted by HFSS and the inset presents the treatment of the discretization of the graded-index metamaterials.

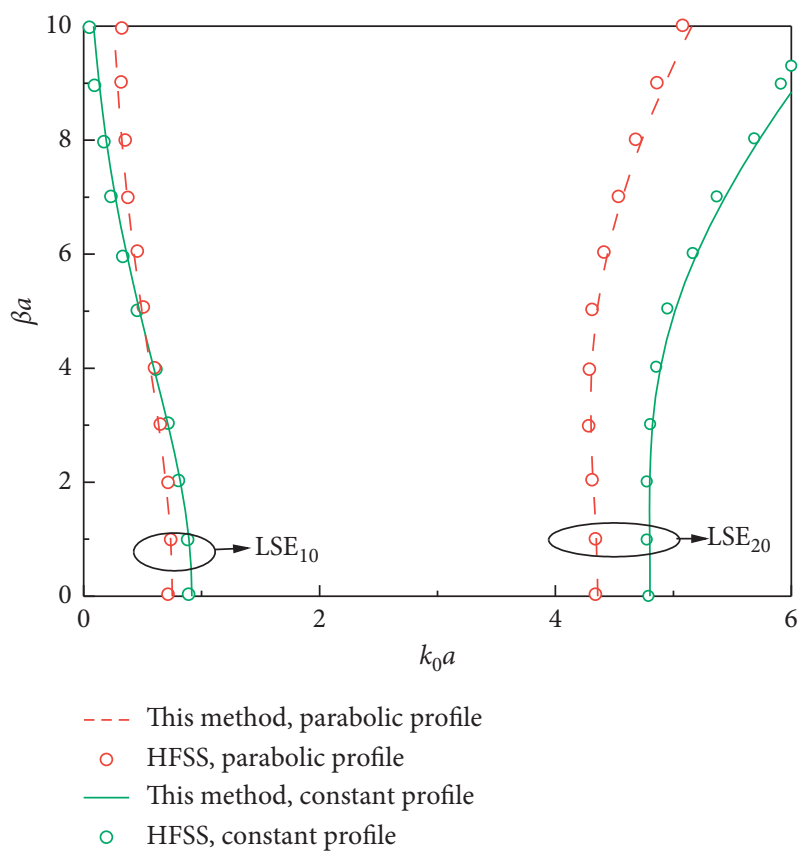

FIgURE 10: Dispersion diagrams of a rectangular waveguide for constant and parabolic profile functions; the curves illustrated with green or red lines are obtained with the proposed technique; curves with green or red circles are obtained with HFSS simulation.

discretization of the graded-index metamaterials is ten subregions, and convergence to $0.1 \%$ on $\Delta \beta$ is achieved in 7 steps (using the eigenmode simulation).
TABLE 1: Computation time comparison of the semianalytical method and the HFSS simulation.

\begin{tabular}{lcccc}
\hline & \multicolumn{2}{c}{$\begin{array}{c}\text { Semianalytical } \\
\text { method }\end{array}$} & \multicolumn{2}{c}{ HFSS simulation } \\
& LSE $_{10}$ & LSE $_{20}$ & LSE $_{10}(\mathrm{~s})$ & LSE $_{20}(\mathrm{~s})$ \\
\hline Constant profile & 15 & 37 & 5.6 & 6.2 \\
Parabolic profile & 42 & 1.2 & 23.4 & 25.9 \\
\hline
\end{tabular}

Compared with the results obtained by the proposed numerical, the convergent results of the propagation constants for the graded-index metamaterials are compared. The dispersion diagrams for constant and linear dielectric profile functions are proposed in Figure 10, and the results agree well with each other.

Although the comparisons between the proposed numerical and the HFSS agree well with each other, the consumed calculation time is very different. In this paper, the code of the proposed method and the HFSS simulation are both performed by using a regular $\mathrm{PC}$ with an Intel Corei7-8500 CPU at $1.80 \mathrm{GHz}$ (eight CPUs) and $16 \mathrm{~GB}$ of RAM for rectangular waveguide filled with graded-index CRLHM. The computation time comparison of the proposed method and the HFSS simulation is presented in Table 1. 30 points of $\beta$ have been computed in both methods. From Table 1, for constant profile function, the calculation time of the proposed technique needs overall tens of seconds to compute the eigenvalues, while the HFSS simulation time is about five minutes. For parabolic profile functions, not considering the time-consuming of 
the convergence analysis, the HFSS simulation time is up to twenty minutes, while the calculation time of the proposed techniques only takes up to nearly one minute. Therefore, the numerical method proposed in this paper has both accuracy and efficiency.

\section{Conclusion}

In this paper, an accurate semianalytical modal theory for propagation in inhomogeneous waveguides has been developed for rectangular waveguides filled with graded-index metamaterials. Based on this theory, the cutoff frequencies and the dispersion relations have been computed for waveguides with various graded-index profile functions. These results have been validated by comparison with the results obtained by the commercial simulation software HFSS. It should be noted that HFSS cannot set the spatially dependent dielectric parameters, and the discretization and convergence analysis must be conducted in the simulation of graded-index metamaterials. The technique proposed in this paper can be employed for arbitrary inhomogeneity analysis. Moreover, the proposed technique is far more efficient than the HFSS simulation. Finally, exciting phenomena of such waveguides containing composite LHM and RHM with graded-index profiles have been highlighted. By choosing appropriate profile functions, the lower cutoff frequency might be substantially reduced compared with classical waveguides, and backward wave propagation below the cutoff frequency is obtained, which allows waveguide miniaturization. The losses and conductivity of the metamaterial are not considered in this paper. The modal theory presented in this paper is still verified if the losses and conductivity are known. Further work is related to the specific implementation of the graded-index metamaterials with consideration of dispersion and losses properties. It has been experimentally verified that a linear graded-index metamaterial could be realized by an array of split-ring resonators (SRRs) with a linearly varying substrate depth, and the graded permeability might be realized by arranging a set of wires that gradually changes their density. The technique may be easily applied to the circular waveguides, the elliptical waveguides, and the polygonal waveguides, by replacing the trigonometry functions in potential functions into the Bessel functions and Mathieu functions.

\section{Data Availability}

The data used to support the findings of this study are included within the article.

\section{Conflicts of Interest}

The authors declare that they have no conflicts of interest.

\section{Acknowledgments}

This research was funded by the Leading Talents of the Guangdong Province Program, grant no. 2016LJ06D557 and the AoShan Talents Outstanding Scientist Program
Supported by the Pilot National Laboratory for Marine Science and Technology (Qingdao), grant no. 2017ASTCPOS03.

\section{References}

[1] M. M. M. Ali, S. I. Shams, A. Sebak, and A. A. Kishk, "Rectangular waveguide cross-guide couplers: accurate model for full-band operation," Institute of Electrical and Electronics Engineers Microwave and Wireless Components Letters, vol. 28, no. 7, pp. 561-563, 2018.

[2] M. Bozzi, A. Georgiadis, and K. Wu, "Review of substrateintegrated waveguide circuits and antennas," IET Microwaves, Antennas \& Propagation, vol. 5, no. 8, pp. 909-920, 2011.

[3] A. Buesa-Zubiria and J. Esteban, "Design of five-way Bagley polygon power dividers in rectangular waveguide," Institute of Electrical and Electronics Engineers Transactions on Microwave Theory and Techniques, vol. 66, no. 1, pp. 116-127, 2017.

[4] W.-K. Cao, L.-T. Wu, C. Zhang et al., "Asymmetric transmission of acoustic waves in a waveguide via gradient index metamaterials," Science Bulletin, vol. 64, no. 12, pp. 808-813, 2019.

[5] R. E. Collin, Field Theory of Guided Waves, IEEE Press, New York, NY, USA, 2nd edition, 1991.

[6] C. Carceller, P. Soto, V. E. Boria et al., "Design of hybrid folded rectangular waveguide filters with transmission zeros below the passband," Institute of Electrical and Electronics Engineers Transactions on Microwave Theory and Techniques, vol. 64, no. 2, pp. 475-485, 2016.

[7] M. Dalarsson and P. Tassin, "Analytical solution for wave propagation through a graded index interface between a righthanded and a left-handed material," Optics Express, vol. 17, no. 8, pp. 6747-6752, 2009.

[8] N. Engheta and R. W. Ziolkowski, Metamaterials: Physics and Engineering Explorations, pp. 87-96, John Wiley \& Sons, Hoboken, NJ, USA, 2006.

[9] W. Fang and S. Xu, "New electromagnetic absorbers composed of left-handed and right-handed materials," International Journal of Infrared and Millimeter Waves, vol. 29, no. 8, pp. 799-807, 2008.

[10] T. Itoh, Numerical Techniques for Microwave and MillimeterWave Passive structures, John Wiley \& Sons, Hoboken, NJ, USA, 1989.

[11] J. M. Jin, The Finite Element Method Electromagn, Wiley, Hoboken, NJ, USA, 2015.

[12] L. Kuhler, G. Le Fur, L. Duchesne, and N. Raveu, "The propagation characteristics of 2-D metamaterial waveguides using the modal expansion theory," Institute of Electrical and Electronics Engineers Transactions on Microwave Theory and Techniques, vol. 66, no. 10, pp. 4319-4326, 2018.

[13] F.-Y. Meng, Q. Wu, D. Erni, and L.-W. Li, "Controllable metamaterial-loaded waveguides supporting backward and forward waves," Institute of Electrical and Electronics Engineers Transactions on Antennas and Propagation, vol. 59, no. 9, pp. 3400-3411, 2011.

[14] W. Y. Lee and S. Y. Wang, "Guided-wave characteristics of optical graded-index planar waveguides with metal cladding: a simple analysis method," Journal of Lightwave Technology, vol. 13, no. 3, pp. 416-421, 1995.

[15] D. R. Smith, W. J. Padilla, D. C. Vier, S. C. Nemat-Nasser, and S. Schultz, "Composite medium with simultaneously negative permeability and permittivity," Physical Review Letters, vol. 84 , no. 18 , p. $4184,2000$. 
[16] K. Siakavara, "Modal analysis of the microwave frequency response and composite right-/left-handed operation of a rectangular waveguide loaded with double positive and double negative materials," International Journal of RF and Microwave Computer-Aided Engineering, vol. 1, pp. 435-445, 2010.

[17] D. R. Smith, J. J. Mock, A. F. Starr et al., "Graded-index metamaterials," Physical Review E, vol. 71, no. 3, Article ID 036609, 2005.

[18] A. Weisshaar and V. K. Tripathi, "Modal analysis of step discontinuities in graded-index dielectric slab waveguides," Journal of Lightwave Technology, vol. 10, no. 5, pp. 593-602, 1992.

[19] F. Parment, A. Ghiotto, T.-P. Vuong, J.-M. Duchamp, and $\mathrm{K}$. $\mathrm{Wu}$, "Double dielectric slab-loaded air-filled SIW phase shifters for high-performance millimeter-wave integration," IEEE Transactions on Microwave Theory and Techniques, vol. 64, no. 9, pp. 2833-2842, 2016. 